Gabriel Dumouchel

Université de Montréal (Canada)

Thierry Karsenti

Université de Montréal (Canada)

\section{Comment les futurs enseignants du Québec évaluent l'information trouvée sur le Web : une étude des pratiques déclarées et effectives}

How do Quebec preservice teachers evaluate information found online: a study of declared and effective practices

doi: 10.18162/fp.2019.449

\section{ésumé}

Cette étude a pour objectif de décrire et analyser les pratiques déclarées et effectives des futurs enseignants du Québec en évaluation de l'information sur le Web. En analysant ces pratiques par l'entremise d'un questionnaire et d'exercices de recherche d'information sur le Web, nos résultats montrent qu'ils sont majoritairement critiques face à l'information qu'ils trouvent sur le Web et qu'ils l'évaluent avec une bonne diversité de critères. Les résultats sont discutés à la lumière de la littérature et des réalités du terrain afin d'offrir des pistes de recommandations pour améliorer le développement de ces compétences en formation initiale.

\section{Mots-clés \\ Évaluation de l'information, traitement de l'information, formation initiale, futurs enseignants, Québec.}

Abstract

The purpose of this study is to describe and analyze the actual and declared practices of Quebec preservice teachers in evaluating information found on the Web. These practices were assessed using a survey and real-time Web research exercises. Our results show that most preservice teachers are critical of information found on the Web and evaluate it using a range of criteria. These findings are discussed in light of the literature and the realities that teachers face in schools, based on which recommendations are made to enhance the development of these competencies in initial training.

Keywords

Evaluation of information, information processing, initial training, preservice teachers, Quebec.
Depuis plus d'une décennie, la très grande majorité des élèves passent en premier lieu par le Web et, plus particulièrement par Google, pour chercher de l'information dans le cadre de leur formation (Case, 2012). Cette réalité représente cependant des défis majeurs en termes d'évaluation de l'information. De fait, en allant en premier lieu sur le Web, l'apprenant n'est plus face à un corpus d'informations triées sur le volet par des spécialistes de la documentation ou automatiquement validées en amont par ses enseignants (Serres, 2005). Parallèlement, il doit aussi apprendre à reconnaître et à éviter diverses infopollutions (Sutter, 1998), comme la désinformation et l'information référencée en priorité dans les résultats de recherche d'un moteur comme Google, ce qui remet en question la pertinence réelle des informations trouvées (Peres, 2015). Par conséquent, dans son nouveau rapport à l'information, l'élève doit faire preuve d'autonomie, de responsabilité, de jugement et d'éthique (Tardif, 1998). De son côté, l'enseignant doit troquer son rôle de transmetteur encyclopédique unique pour celui de médiateur et de guide (Damour, 2013) et amener l'élève à développer ses compétences en évaluation de l'information.

De ce fait, au Québec comme ailleurs, divers référentiels de formation des enseignants précisent qu'ils doivent être en mesure de savoir évaluer l'information trouvée sur Internet (International Society for Technology in Education, 2008; Martinet, Raymond et Gauthier, 2001; Ministère de l'Enseignement supérieur et de la Recherche, 2011). Certes, ces compétences sont considérées comme étant essentielles pour les enseignants actuels et futurs, mais la littérature démontre que plusieurs présentent des lacunes à ce sujet. De ce fait, selon une étude internationale réalisée auprès de 35000 enseignants du secondaire (Fraillon, Ainley, Schulz, Friedman et Gebhardt, 2014), $92 \%$ d'entre eux ont affirmé se sentir compétents pour trouver des ressources utiles pour leur enseignement sur le Web. Toutefois, à peine la moitié de ces mêmes répondants ont signalé enseigner à leurs 
élèves comment évaluer la pertinence, la crédibilité et l'exactitude de l'information trouvée sur Internet. De son côté, en sondant 19 enseignants québécois, Gervais (2011) a constaté que seul le tiers d'entre eux effectuaient des activités d'apprentissage liées à l'évaluation de l'information avec leurs élèves.

Pour leur part, les futurs enseignants sont nombreux à trouver très important de savoir juger de la crédibilité de l'information trouvée sur Internet (Giroux, Gagnon, Gremion et Heinzen, 2014; Lefebvre, 2014). Toutefois, s'ils se disent fort compétents pour évaluer l'information (Simard et Karsenti, 2016; Stockham et Collins, 2012), il reste que de nombreuses études démontrent que leurs compétences réelles sont plutôt lacunaires puisqu'ils utilisent peu de critères quand ils doivent faire l'évaluation d'une information trouvée sur le Web (Eret et Ok, 2014; Giroux, Gagnon, Lessard et Cornut, 2011).

En somme, les futurs enseignants actuels reconnaissent l'importance de savoir évaluer l'information trouvée sur le Web et se voient majoritairement comme étant très compétents pour le faire. Cependant, les recherches démontrent plutôt qu'ils maîtrisent encore insuffisamment ces compétences, bref que la formation reçue ne semble pas apporter les fruits espérés. Afin de mieux comprendre en quoi consiste plus précisément la compétence en évaluation de l'information, les deux prochaines sections s'attardent à la définir.

\section{L'évaluation de l'information : une procédure basée sur des critères multiples et souvent flous}

Il existe plusieurs dizaines de critères permettant d'évaluer une information trouvée sur le Web, certains touchant soit son utilité pour répondre à un besoin d'information, son contenu, son format, sa présentation, sa provenance ou encore les efforts requis pour l'obtenir (Boubée et Tricot, 2010). Or, selon Serres (2012), certains critères comme la crédibilité et l'autorité sont souvent enchevêtrés. De plus, sur le Web, une information peut être produite par plusieurs auteurs souvent anonymes et être modifiée sans préavis, notamment sur Wikipédia. Bref, la multitude de critères d'évaluation, le flou conceptuel de certains d'entre eux et la nature mouvante de l'écosystème informationnel compliquent la tâche des élèves comme des enseignants pour savoir comment bien évaluer une information trouvée sur le Web. Par contre, Boubée et Tricot (2010) soulignent qu'environ dix critères sont habituellement employés pour évaluer une information trouvée sur le Web et qu'il n'existe pas vraiment de hiérarchie des critères puisque leur choix dépend de divers éléments comme le contexte ou le temps disponible pour effectuer une recherche. Par conséquent, dans le cadre de notre étude, nous avons adapté et regroupé divers critères d'évaluation recensés par plusieurs auteurs (Boubée et Tricot, 2010; Broudoux, 2007; Hjorland, 2012; Karsenti, Dumouchel et Komis, 2014; Metzger, 2007; Serres, 2012) que nous décrivons dans le tableau 1. Par ailleurs, étant donné que notre étude porte sur les futurs enseignants, des codes relatifs à l'évaluation de l'information à des fins d'enseignement et d'apprentissage ont été ajoutés à ces derniers. 


\section{Tableau 1}

Critères d'évaluation de l'information trouvée sur le Web.

\begin{tabular}{|c|c|}
\hline Critère & Définition \\
\hline 1. Actualité de l'information & $\begin{array}{l}\text { La date et/ou la mise à jour de la publication ou diffusion d'une information trouvée sont-elles } \\
\text { récentes? }\end{array}$ \\
\hline 2. Autorité de contenu & $\begin{array}{l}\text { Le type de contenu fait autorité. C'est le cas notamment de l'autorité conférée aux livres et aux } \\
\text { articles scientifiques. }\end{array}$ \\
\hline 3. Crédibilité de surface & $\begin{array}{l}\text { Qualité de la mise en forme (p. ex., couleurs, graphisme) ou de la langue (p. ex., orthographe, } \\
\text { grammaire, syntaxe) de l'information trouvée. }\end{array}$ \\
\hline 4. Crédibilité réputée & $\begin{array}{l}\text { L'information trouvée est évaluée selon sa réputation dans un domaine (p. ex., une information } \\
\text { gouvernementale versus celle provenant d'un wiki ou du site Web d'un quidam). }\end{array}$ \\
\hline 5. Exhaustivité & L'information trouvée est-elle détaillée et complète? \\
\hline 6. Expertise de l'auteur & L'information est évaluée selon l'expertise et la formation de son auteur. \\
\hline 7. Identification de l'auteur & L'auteur d'une information trouvée est identifiable. \\
\hline 8. Intérêt & L'information trouvée est intéressante. \\
\hline $\begin{array}{l}\text { 9. Pertinence à des fins } \\
\text { d'enseignement }\end{array}$ & Pertinence de l'information trouvée pour être utilisée à des fins d'enseignement. \\
\hline $\begin{array}{l}\text { 10. Pertinence à des fins } \\
\text { d'apprentissage }\end{array}$ & $\begin{array}{l}\text { Pertinence de l'information trouvée en regard de son adaptation au niveau scolaire des élèves } \\
\text { (p. ex., adaptée pour ceux du primaire ou du secondaire). }\end{array}$ \\
\hline $\begin{array}{l}\text { 11. Pertinence de l'effort } \\
\text { d'obtention }\end{array}$ & Pertinence d'une information selon l'effort qu'elle requiert pour être obtenue (p. ex., temps requis). \\
\hline $\begin{array}{l}\text { 12. Pertinence envers le besoin } \\
\text { d'information }\end{array}$ & L'information trouvée permet-elle de répondre au besoin d'information établi? \\
\hline 13. Références bibliographiques & L'information trouvée comporte des références bibliographiques. \\
\hline 14. Synthèse & L'information trouvée est présentée de manière synthétisée. \\
\hline $\begin{array}{l}\text { 15. Validation sociale } \\
\text { de l'information }\end{array}$ & $\begin{array}{l}\text { L'information est validée par des pairs consultés personnellement } \\
\text { (p. ex., experts, collègues, amis, famille). }\end{array}$ \\
\hline 16. Vérifiabilité & L'information trouvée peut-elle être comparée avec d'autres qui l'appuient ou l'infirment? \\
\hline
\end{tabular}




\section{Objectif de recherche}

Cette étude a pour objectif de décrire et analyser les pratiques déclarées et effectives des futurs enseignants en évaluation de l'information sur le Web. La prochaine section détaille les aspects méthodologiques de cette étude.

\section{Méthodologie}

Les outils de collecte et d'analyse de cette étude ont été élaborés selon le processus de recherche et de traitement del'information préconisé par divers modèles dominants relativement similaires (Association of College and Research Libraries, 2000; Eisenberg et Berkowitz, 1990; Kuhlthau, 1993; Marchionini, 1995). Cependant, dans le cadre du présent article, nous nous en tiendrons uniquement à l'évaluation de l'information. Ainsi, nous nous intéressons aux critères employés par les futurs enseignants pour évaluer l'information qu'ils trouvent sur le Web (voir tableau 1).

\section{Participants}

Notre étude a été réalisée au cours de l'année universitaire 2012-2013 auprès de futurs enseignants issus des différents baccalauréats en enseignement de la Faculté des sciences de l'éducation de l'Université de Montréal (UdeM; n = 2137).

\section{Collecte des données}

Afin de trianguler nos données, deux outils de collecte complémentaires ont été mis à contribution dans le cadre de cette étude, à savoir un questionnaire et des exercices de recherche et de traitement de l'information sur le Web.

Dans un premier temps, le questionnaire comprenait une question ouverte demandant aux répondants d'expliquer comment ils vérifient la crédibilité de l'information trouvée sur Internet. Sur les 2137 répondants potentiels provenant des programmes de baccalauréat en enseignement de l'UdeM ${ }^{1}$, 353 futurs enseignants ont dûment rempli le questionnaire, ce qui représente un taux de réponse de $16,5 \%$. En ce qui a trait au genre, 84,7 \% des répondants étaient des femmes contre 15,3\% d'hommes. Les participants étaient principalement âgés de 19 à 24 ans (71,4\%) alors que les autres tranches d'âge étaient de $11,9 \%$ pour les $25-29$ ans, $11,6 \%$ pour les $30-39$ ans et 5,1\% pour les 40 ans et plus. D'autre part, nous constatons une distribution relativement partagée des répondants pour ce qui est de l'année de formation universitaire : $1^{\text {re }}$ année $(26,9 \%), 2^{\mathrm{e}}$ année $(27,8 \%), 3^{\mathrm{e}}$ année $(18,1 \%)$, $4^{\text {e }}$ année $(20,7 \%)$ en plus d'étudiants ayant terminé leur baccalauréat en date de la récolte des données $(6,5 \%)$. Les répondants provenaient de divers programmes de formation, plus particulièrement du baccalauréat en éducation préscolaire et enseignement primaire (BEPEP; 35,5 \%) et du programme d'enseignement en adaptation scolaire $(25,2 \%)$.

Dans un deuxième temps, des exercices de recherche et de traitement de l'information sur le Web ont été élaborés à partir de la méthodologie de Fournier (2007). À cette fin, nous avons fourni un ordinateur portable à neuf volontaires finissants du BEPEP. D'autre part, la grande majorité des neuf participants étaient des femmes $(\sim 78 \%)$, ce qui représente des proportions de genre relativement similaires aux répondants au questionnaire. 
Dans le cadre de ces exercices, les participants devaient d'abord remplir une fiche documentaire sur les changements climatiques adressée à des élèves du primaire afin de les aider à mieux comprendre les grandes lignes de ce thème et à démarrer un projet de recherche à ce sujet. Ils devaient ensuite remplir une seconde fiche documentaire adressée à un enseignant en décrivant divers éléments liés aux tableaux blancs interactifs en enseignement préscolaire-primaire (p. ex., avantages, défis) et en proposant des activités avec cet outil. Un logiciel de capture d'écran a permis d'enregistrer les actions de recherche et de traitement de l'information des participants en plus de leurs réflexions orales sur leurs faits et gestes. Il est à noter que les participants ont effectué ces exercices sur un ordinateur portable Mac fourni par un centre de recherche, une mesure visant à standardiser l'environnement de travail de manière à ce que chaque participant ait eu accès aux mêmes conditions de recherche et de traitement de l'information.

Par ailleurs, nous tenons enfin à souligner que l'utilisation de ces deux outils de collecte avait aussi pour but d'obtenir des données relatives aux pratiques déclarées et effectives des futurs enseignants telles que définies par Maubant (2007). Nous entendons par pratiques déclarées celles qui sont décrites et explicitées par nos participants dans le questionnaire. Quant aux pratiques effectives, elles ont été recueillies par observation, d'où l'emploi dans notre étude des exercices de recherche et de traitement de l'information sur le Web.

\section{Analyse des données}

Les données ont été analysées selon des grilles de codage basées sur notre modèle synthèse de recherche et de traitement de l'information. Des statistiques descriptives et inférentielles ont été obtenues avec le logiciel SPSS 21 à partir des données quantitatives du questionnaire. Des analyses de contenu ont été effectuées avec les données qualitatives au sein de deux logiciels. D'une part, nous avons utilisé le logiciel QDA Miner 4 pour analyser les données recueillies dans la question ouverte du questionnaire ainsi que les fiches produites durant les exercices. Quant aux actions et réflexions vidéographiées des participants aux exercices de recherche d'information sur le Web, nous en avons fait une analyse de contenu avec le logiciel NVivo 10. Afin de nous assurer de la fidélité de nos analyses, nous avons procédé à un contre-codage de $20 \%$ de nos données dans QDA Miner par un second codeur. Cela nous a permis d'obtenir un des taux d'accord interjuge de $79 \%$ pour les questions ouvertes et de $82 \%$ pour les fiches, des taux considérés comme étant satisfaisants par Miles et Huberman (2003). Pour ce qui est des quelque 12 heures d'exercices vidéographiés codées dans NVivo, l'ensemble des actions (données vidéo) et des réflexions (données audio) des participants aux exercices ont été codées par trois personnes selon une approche de validation des données inspirée de Van der Maren (1996). Un premier codeur a d'abord codé $20 \%$ du corpus audiovisuel qu'un second codeur a par la suite contrecodé pour en arriver à un accord interjuge relativement satisfaisant de $62 \%$. Une révision commune de la grille a ensuite été effectuée en vue de l'ajuster afin de permettre au premier codeur de procéder au codage de l'entièreté des données dans NVivo. Enfin, un troisième codeur - le premier auteur de cette étude - a revu l'ensemble du codage dans le but de le raffiner, ce qui dénote un processus d'analyse fort minutieux, collaboratif et complémentaire face à un corpus vidéographié à la fois imposant et riche. 


\section{Résultats}

Dans le cadre de notre étude, nous avons recueilli des données touchant à la manière dont les futurs enseignants évaluent de l'information trouvée sur le Web, et ce, qu'elles émanent de pratiques déclarées (questionnaire) ou effectives (exercices).

\section{Pratiques déclarées d'évaluation de l'information}

Par l'entremise d'une question ouverte, nous avons d'abord demandé aux répondants du questionnaire ( $\mathrm{n}=353)$ comment ils vérifiaient que l'information trouvée sur le Web était crédible (voir figure 1). Ce faisant, neuf principaux critères ont été relevés, dont celui de la vérifiabilité ( $n=161)$, c'est-à-dire le fait de comparer une information avec d'autres, qui a dominé leurs réponses comme en témoigne celle de $\mathrm{Q} 039^{2}$ : «Je regarde les infos puis je compare avec ce que j’ai trourvé ailleurs, ce qui se répète le plus est alors jugé crédible. »

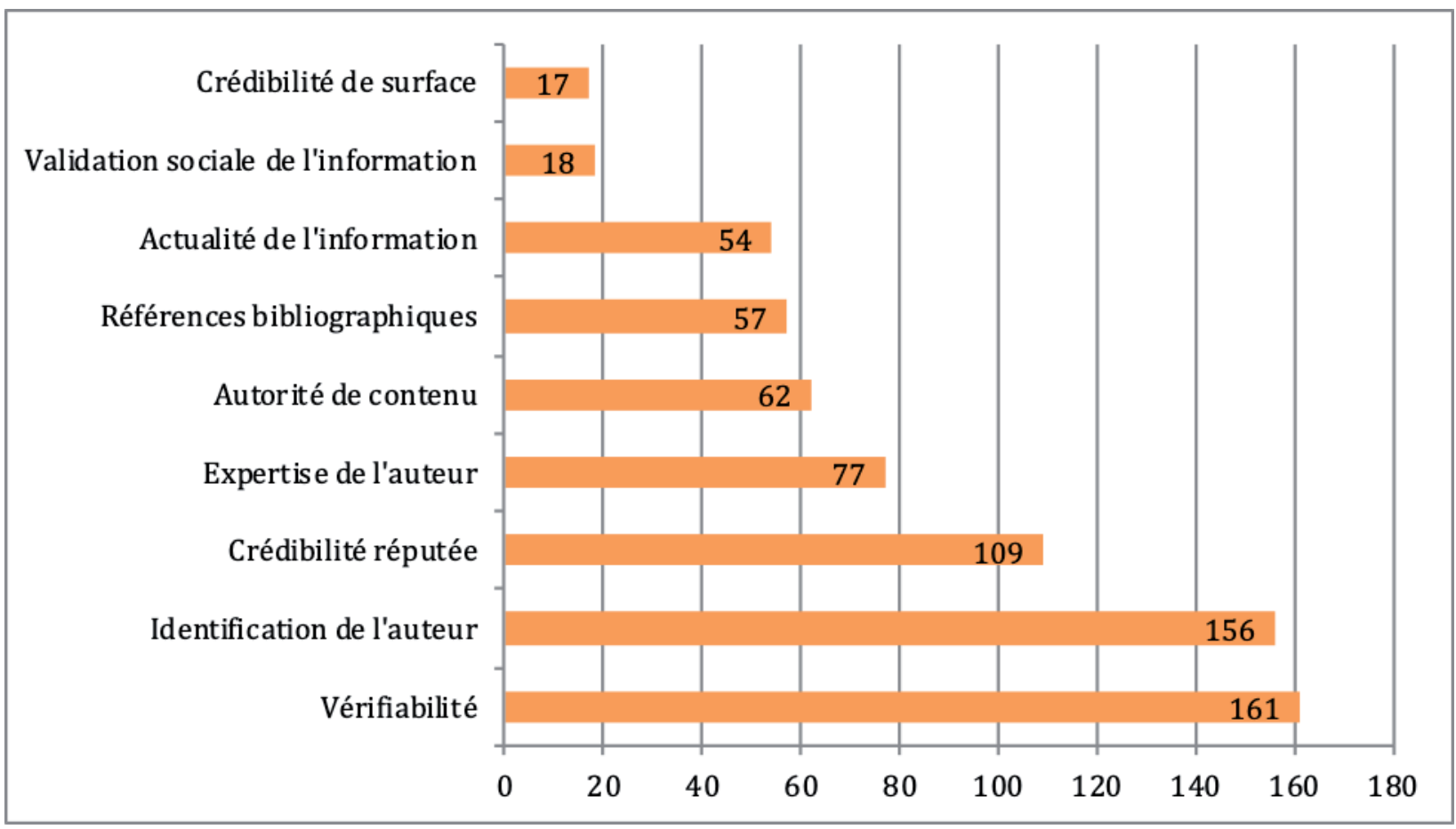

Figure 1

Fréquence des critères donnés par les répondants pour vérifier la crédibilité d'une information trouvée sur le Web.

Deuxièmement, les répondants ont signalé chercher très souvent à identifier l'auteur d'une information trouvée sur le Web $(\mathrm{n}=156)$ : "Si c'est anonyme, je me méfe et je prends l'information avec un grain de sel»(Q202).

Troisièmement, les répondants ont mentionné des aspects relatifs à la crédibilité réputée d'une information $(\mathrm{n}=109)$ : "Je regarde le site Internet à savoir si c'est une association reconnue ou un site gouvernemental»(Q132). 
Parallèlement, le quatrième critère le plus fréquemment relevé est l'expertise de l'auteur $(\mathrm{n}=77)$ : «Je cherche l'auteur de l'article pour voir son parcours scolaire ou de travail»(Q017).

Les répondants ont cinquièmement affirmé se fier à l'autorité de contenu en priorisant certains types de documents comme les livres, les revues scientifiques, les bases de données et les ouvrages de référence (n =62), et ce, parfois en format imprimé : "Sij'ai des doutes sur la validité de certaines informations, je me dirigerai vers des éditions d'ouvrages papier que je considère fiables à la bibliothèque »(Q149).

En sixième position, les répondants signalent vérifier les références bibliographiques dans l'information trouvée sur le Web $(\mathrm{n}=57)$ : "Je vérifie aussi les bibliographies pour être certaine que les sources sont bonnes " (Q257).

Septièmement, les répondants déclarent vérifier l'actualité de l'information trouvée, soit en cherchant la date de publication ou de diffusion, soit la date de mise à jour $(\mathrm{n}=54)$ : «Je regarde le moment quand la dernière mise à jour a été faite. Je privilégie un site encore actif à un site dont les informations sont stagnantes depuis plusieurs années» (Q151).

Enfin, soulignons que d'autres éléments servant à évaluer la crédibilité de l'information ont aussi été mentionnés plus rarement par les répondants, comme de faire valider socialement l'information trouvée, notamment par un professeur, un collègue, un membre de la famille, un ami ou même un internaute lambda $(\mathrm{n}=18)$.

\section{Pratiques effectives en évaluation de l'information}

Nous avons aussi analysé comment neuf futurs enseignants ont évalué l'information selon leurs réflexions orales enregistrées dans le cadre d'exercices de recherche et de traitement d'information sur le Web (voir figure 2). Il en ressort que la majorité a employé au moins neuf des seize critères à cette fin, et que le critère qui est revenu le plus souvent touche la pertinence d'une information trouvée à des fins d'enseignement $(n=71)$. Autrement dit, ces futurs enseignants évaluaient, en premier lieu, si une information pouvait servir en classe et, si oui, comment. C'est ce que le participant V0013 indique quand il affirme : «Je crois que ça pourrait être une bonne idée de déclencheur pour une activité avec les élèves. »

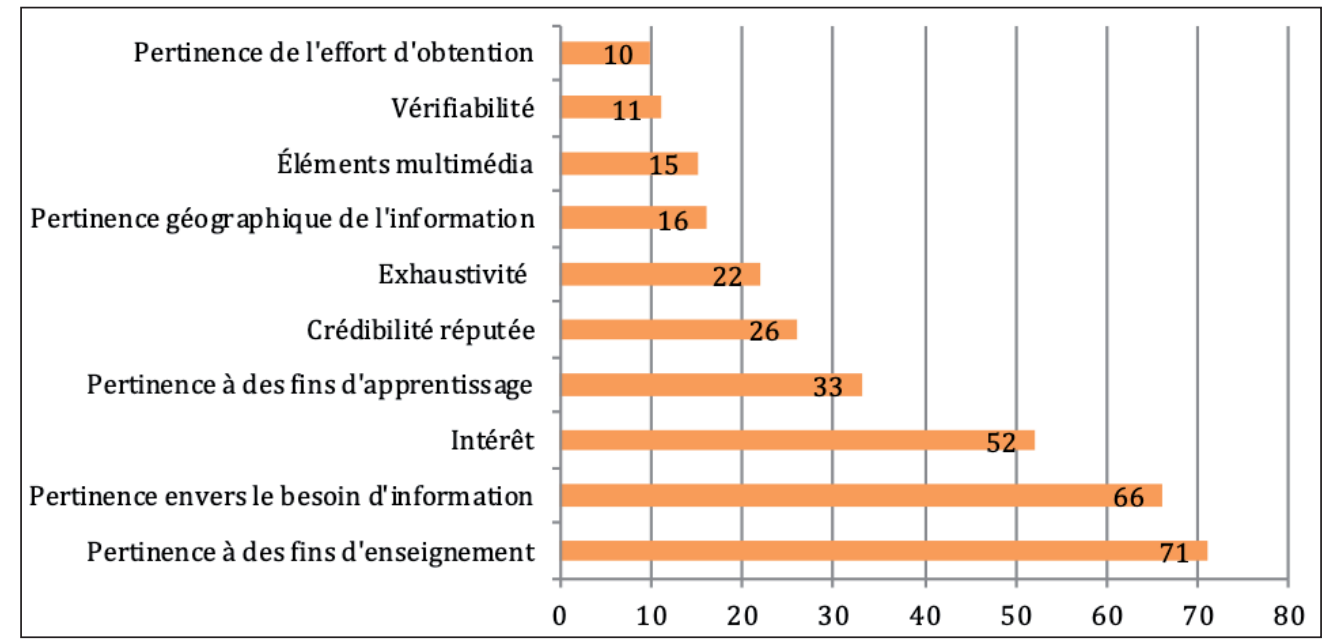

Figure 2

Fréquence des critères d'évaluation signalés oralement par les participants durant les exercices de recherche et de traitement de l'information sur le Web. 
Le deuxième critère le plus récurrent est lié à la pertinence de l'information trouvée en regard du besoin d'information des participants: "Je remarque quen écrivant changement climatique, ça ne me donne pas nécessairement des liens pertinents. On me parle plutôt des actions du Canada sur les changements climatiques»(V002).

Les participants ont troisièmement évalué l'information selon qu'elle était intéressante ou non $(\mathrm{n}=52)$ : "Prise en main d'un tableau interactif... Ça pourrait peut-être être intéressant... Ah ça va être intéressant! C'est exactement le tableau qu'il y avait dans ma classe de stage » (V001).

Quatrièmement, les participants ont vérifié si l'information trouvée était pertinente à des fins d'apprentissage, notamment selon si le contenu était adapté au niveau des élèves $(n=33)$ : "Donc je peux remarquer que le vocabulaire est probablement trop avancé pour les élèves au primaire. Donc si j’utilise ce site Internet là, je vais devoir modifier mon vocabulaire pour l'adapter»(V002).

La crédibilité réputée de l'information trouvée vient quant à elle en cinquième position chez les participants aux exercices de recherche $(\mathrm{n}=26)$ : «Ok, ce quiest le changement climatique... parfait, donc le ministère de l'Environnement, ça l'air d'être certainement un bon site»(V009).

Sixièmement, les participants ont indiqué l'exhaustivité de l'information trouvée $(n=22)$ : «Tableaublancinteractif.fr, histoire TBI, intérêts pédagogiques, les apports, les limites... tout est là » (V008).

En septième position, les participants ont évalué l'information selon son origine géographique $(\mathrm{n}=16)$ : "Étant donné quion est en Amérique du Nord, c'est peut-être plus à propos pour mes élèves " (V002).

Ensuite, dans une moindre mesure, certains participants ont aussi signalé utiliser d'autres critères pour évaluer l'information qu'ils trouvaient sur le Web. Ainsi, presque tous les participants ont indiqué évaluer selon la qualité ou la quantité d'éléments multimédias trouvés $(\mathrm{n}=15)$. Plus de la moitié ont comparé l'information trouvée avec d'autres sources $(n=11)$ alors que quatre des participants l'ont évaluée selon le temps requis pour la consulter $(n=10)$.

\section{Discussion}

Pour rappel, cette étude vise à décrire et analyser les pratiques déclarées et effectives des futurs enseignants en évaluation de l'information sur le Web. Dans cette section, nous discutons des résultats obtenus à la lumière de l'atteinte de cet objectif et de la littérature connexe.

Pour évaluer de l'information trouvée sur le Web, sept critères ont été mentionnés plus de 50 fois par les répondants du questionnaire. Le principal critère mentionné fut de loin la vérifiabilité de l'information. Bien que ce critère soit utile pour évaluer une information, Simonnot (2007) souligne qu'il est limité par la difficulté à retrouver la source originale puisque le Web est peuplé d'informations redondantes, une conséquence directe de la reprise, de la copie, de la rediffusion ou encore de l'extraction hors contexte de l'information. Ce recours à la comparaison d'informations peut cependant être mis en relation avec un autre critère qui a souvent été cité par les répondants, à savoir l'autorité de contenu, plus précisément une plus grande confiance envers des documents comme les livres et les revues scientifiques. D'ailleurs, de nombreuses études (Béguin-Verbrugge et Kovacs, 2011; Sahut, 2014; Watson, 2014) ont relevé 
l'importance de ce critère et plusieurs de nos répondants ont signalé évaluer l'information en allant comparer dans des documents de ce type, notamment sous format papier.

D'autre part, les deuxième, troisième et quatrième critères les plus souvent mentionnés par les répondants sont complémentaires puisqu'ils touchent tous l'auteur d'une information. De ce fait, les répondants affirment chercher à identifier l'auteur pour ensuite vérifier son affiliation (crédibilité réputée) ainsi que son expertise au point de vue de sa formation et de ses réalisations. Certains ont d'ailleurs signalé délaisser une information s'ils n'arrivaient pas à identifier son auteur, si elle provenait d'un site personnel ou d'un wiki ou encore si son auteur n'était pas un expert dans son domaine. Cette propension à vouloir évaluer l'information selon ces trois critères est encourageante si on la compare aux résultats obtenus par d'autres études à ce sujet où les futurs enseignants n'y faisaient pas appel (voir Hogan et Varnhagen, 2012).

De leur côté, les futurs enseignants qui ont participé aux exercices de recherche vidéographiés ont eu recours à un attirail de critères quelque peu différents pour évaluer l'information trouvée sur le Web. Ainsi, ils ont majoritairement signalé évaluer l'information selon la manière dont elle pouvait être utilisée à des fins d'enseignement et d'apprentissage, une pratique d'ailleurs relevée par d'autres recherches auprès de futurs enseignants (Beach et Willows, 2014; Uppal, 2011). Alors que ces critères sont fort utiles pour enseigner, ils ne se retrouvent pas dans les principaux critères mentionnés par les répondants. Cette absence peut notamment s'expliquer par le besoin d'information prescrit à nos participants par l'entremise de nos outils de collecte de données de recherche. De fait, en cherchant et évaluant de l'information en temps réel sur le Web dans le cadre des exercices, les participants étaient exposés à une mise en situation qui leur demandait expressément de produire une fiche d'information pour des élèves. Le questionnaire demandait plutôt aux futurs enseignants ce qu'ils utilisaient comme critères pour évaluer de l'information pour leurs besoins en formation initiale. Il se peut donc que les répondants aient davantage réfléchi aux critères employés pour trouver de l'information qu'ils réinvestissent dans leurs travaux universitaires plutôt qu'en situation d'enseignement. En somme, nos résultats confirment que le choix des critères d'évaluation d'information sur le Web des futurs enseignants est largement influencé par le besoin d'information à combler.

D'autre part, qu'il s'agisse des critères d'évaluation déclarés par les répondants au questionnaire ou ceux utilisés effectivement par les participants aux exercices, il demeure que le nombre de critères utilisés par la majorité de nos participants est fort éloigné du 1,34 critère d'évaluation utilisé par les futurs enseignants québécois de l'étude de Giroux et al. (2011). De fait, nous constatons que la majorité des participants aux exercices de recherche vidéographiés ont utilisé au moins neuf critères pour évaluer l'information trouvée sur le Web. De leur côté, sept critères ont été mentionnés par plus de 50 répondants au questionnaire. Ce large écart dans le nombre de critères entre les résultats de notre étude et ceux de Giroux et al. (2011) pourrait s'expliquer en partie par leur méthodologie de recherche. En effet, leurs participants ont peut-être sciemment choisi de limiter leur nombre de critères pour évaluer des sites Web puisqu'ils en avaient 300 à analyser. 


\section{Forces et limites de la recherche}

Notre étude comporte diverses forces et limites qu'il importe de souligner. Du côté des forces, notons sa méthodologie mixte (Van der Maren, 1996) et novatrice avec l'analyse des pratiques effectives par l'entremise de données vidéographiées. Il s'agit aussi d'une des rares recherches ayant étudié la mise en pratique des compétences informationnelles des futurs enseignants québécois sur le Web (Fournier, 2007; Gervais, 2004). Elle permet de plus de constater certaines lacunes rencontrées tout en offrant des pistes de solution à cet effet. Quant aux limites de cette recherche, notons d'une part que les résultats obtenus se basent sur un échantillon d'étudiants d'une seule université québécoise et qu'ils ne peuvent donc pas être généralisés à l'ensemble des futurs enseignants du Québec. D'autre part, les participants aux exercices de recherche vidéographiés nont pas toujours expliqué en détail les raisons pour lesquelles ils considéraient une information trouvée comme étant crédible ou non.

\section{Pistes de recherches}

Parallèlement, de nouvelles recherches pourraient demander aux participants de visionner leurs exercices vidéographiés afin qu'ils puissent commenter et clarifier leurs actions. Il serait aussi plus adéquat que les futurs enseignants puissent utiliser leur propre outil technologique dans le cadre de tels exercices afin qu'ils agissent dans un contexte davantage authentique d'évaluation de l'information. Il serait aussi fort intéressant d'étudier comment les futurs enseignants évaluent l'information trouvée par l'entremise des médias sociaux. À la différence d'une quête d'information qu'ils doivent eux-mêmes effectuer sur un moteur de recherche, ils reçoivent des informations que des « amis » partagent avec eux sur ces réseaux. Quels critères d'évaluation vont-ils alors privilégier alors que la désinformation et les informations référencées qui apparaissent dans les résultats de requête des moteurs de recherche (Peres, 2015) se retrouvent aussi dans les informations diffusées et partagées dans les médias sociaux (Mintz, 2012)? Cet aspect est particulièrement important à étudier avec l'arrivée récente de lère de la post-vérité, où plusieurs personnes considèrent que les émotions et les intérêts personnels ont davantage de poids que les faits dûment vérifiés (Larivée, 2017). De ce fait, les médias sociaux comme Facebook permettent de consolider les opinions et valeurs de leurs usagers en les invitant à se créer un réseau d' « amis » en plus de leur offrir un fil d'information conforme à leurs intérêts, celui-ci étant élaboré à l'aide d'algorithmes déterminant les contenus les plus susceptibles d'intéresser un profil en particulier (Ertzscheid, 2015). Ainsi conçus, ces médias sociaux peuvent en venir à placer des individus dans un circuit d'information fermé - Ertzscheid parle d'enceintes algorithmiques - où règne une vision incomplète et biaisée de la réalité. D'autre part, avec leur pouvoir de viralité et la brièveté des informations qui y sont transmises, ces médias sociaux sont souvent utilisés en tant que vecteurs de faux contenus à des fins politiques ou mercantiles (Allcott et Gentzkow, 2017). La désinformation y est publiée puis partagée à un rythme si rapide que la validation factuelle devient un travail perpétuel digne de Sisyphe. Le problème est devenu si grave que Facebook s'attaque désormais aux fausses nouvelles et à leurs auteurs sévissant sur son réseau (Shaik, 2017). Bref, force est de constater qu'en se limitant simplement aux médias sociaux, la question de l'évaluation de l'information chez les enseignants tant actuels que futurs représente un champ de recherche incontournable et en pleine expansion. 


\section{Conclusion}

Cette étude démontre que les futurs enseignants du Québec sont majoritairement critiques face à l'information qu'ils trouvent sur le Web et qu'ils l'évaluent avec une bonne diversité de critères. Toutefois, on remarque que les critères employés diffèrent selon la nature de leurs pratiques. Récoltées par l'entremise de questionnaires, les pratiques déclarées concernaient des critères s'appliquant davantage à leurs besoins dans des travaux de nature universitaire. De leur côté, les pratiques effectives s'appuyaient principalement sur des aspects pratico-pratiques de l'évaluation de l'information tels que leur utilité à des fins d'enseignement et d'apprentissage. On peut percevoir dans cette différence la dualité informationnelle de leur formation initiale, où l'information évaluée doit servir tant dans leurs cours universitaires que dans leurs stages en enseignement. En quelque sorte, ces futurs enseignants font donc montre d'un pragmatisme dont il faut tenir compte dans le cadre de leur formation.

Parallèlement, les résultats de notre étude nous permettent de faire quelques recommandations à cet effet. Ainsi, nous sommes d'avis que les futurs enseignants doivent être formés à évaluer l'information sur le Web en les confrontant aux infopollutions et en apprenant à en créer à des fins éducatives. Il faut d'une part, les former à déceler les infopollutions afin d'être mieux préparés à enseigner comment les détecter. D'autre part, à l'instar de certains de nos participants, il faut qu'ils maîtrisent certains outils du Web, comme Wikipédia, afin de savoir comment l'information peut être créée puis diffusée et ainsi démontrer à leurs futurs élèves à quel point il importe d'être critique face à l'information qui se trouve sur le Web. Cela revient en quelque sorte à rendre le canular éducatif, car ce n'est pas en leur donnant une liste de critères d'évaluation que les futurs enseignants seront adéquatement formés. Il faut les placer dans des situations authentiques sur le Web où ils doivent se débrouiller et comprendre peu à peu les mécanismes de l'écosystème informationnel dans lequel eux et leurs élèves évoluent. Bref, il importe d'amener les futurs enseignants à être à la fois lucides et prudents pour évaluer et utiliser l'information. Ils seront ainsi mieux préparés pour rendre leurs élèves autonomes et critiques face à cet écosystème mouvant et trop souvent infopollué.

\section{Notes}

1 Baccalauréats en éducation préscolaire et enseignement primaire, en enseignement de l'éducation physique et à la santé, en enseignement de l'éthique et de la culture religieuse au secondaire, en enseignement de l'univers social au secondaire, en enseignement des mathématiques au secondaire, en enseignement des sciences et des technologies au secondaire, en enseignement du français au secondaire, en enseignement du français langue seconde, en enseignement en adaptation scolaire.

2 Ce code représente le numéro du répondant au questionnaire.

3 Ce code représente le numéro du participant aux exercices vidéographiés.

\section{Références}

Allcott, H. et Gentzkow, M. (2017). Social media and fake news in the 2016 election (Working paper $\mathrm{n}^{\circ}$ 23089). Cambridge, MA : National Bureau of Economic Research. http://dx.doi.org/10.3386/w23089

Association of College and Research Libraries (ACRL). (2000). Information literacy competency standards for higher education. Chicago, IL : ACRL. 
Beach, P. et Willows, D. (2014). Investigating teachers' exploration of a professional development website: An innovative approach to understanding the factors that motivate teachers to use Internet-based resources. Revue canadienne de l'apprentissage et de la technologie, 40(3). http://dx.doi.org/10.21432/T2RP47

Béguin-Verbrugge, A. et Kovacs, S. (dir.). (2011). Le cabier et l'écran. Culture informationnelle et premiers apprentissages documentaires. Paris : Hermès.

Boubée, N. et Tricot, A. (2010). Qu'est-ce que rechercher de l'information? Villeurbanne : Presses de l'ENSSIB. http://dx.doi.org/10.4000/books.pressesenssib.799

Broudoux, E. (2007). Construction de l'autorité informationnelle sur le web. Dans R. Skare, N. W. Lund et A. Varheim (dir.), A document (re)turn: Contributions from a research field in transition (p. 1-11). Repéré à https://archivesic.ccsd.cnrs.fr/sic 00120710/document

Case, D. O. (2012). Looking for information: A survey of research on information seeking, needs, and behavior ( $3^{\mathrm{e}}$ éd.). Bingley : Emerald.

Damour, F. (2013). Comment Internet m’a ré-appris pourquoi j’enseigne. Études, 419(9), 173-184.

Eisenberg, M. B. et Berkowitz, R. E. (1990). Information problem-solving: The Big Six Skills approach to library E information skills instruction. Norwood, $\mathrm{NJ}:$ Ablex.

Eret, E. et Ok, A. (2014). Internet plagiarism in higher education: Tendencies, triggering factors and reasons among teacher candidates. Assessment and Evaluation in Higher Education, 39(8), 1002-1016. http://dx.doi.org/10.1080/02602938.2014.880776

Ertzscheid, O. (2015). Usages de l'information numérique : comprendre les nouvelles enclosures algorithmiques pour mieux s'en libérer. Revue française des sciences de l'information et de la communication, (6). http://dx.doi.org/10.4000/rfsic. 1425

Fournier, H. (2007). Stratégies de recherche et de traitement de l'information dans des environnements informatiques et sentiment d'efficacité personnelle des futurs enseignants à l'égard de ces stratégies (Thèse de doctorat, Université du Québec à Montréal). Repéré à https://archipel.uqam.ca/807

Fraillon, J., Ainley, J., Schulz, W., Friedman, T. et Gebhardt, E. (2014). Preparing for life in a digital age: The IEA International Computer and Information Literacy Study international report. Repéré à https://research.acer.edu.au/cgi/viewcontent.cgi?article=1009\&context=ict literacy

Gervais, S. (2004). Les habiletés en recherche d'information des étudiant(e)s universitaires : une observation (Mémoire de maîtrise inédit). Université de Montréal.

Gervais, S. (2011). Accès aux ressources numériques et leur utilisation par les enseignants : résultats d'un sondage. Documentation et bibliothèques, 57(3), 133-152. http://dx.doi.org/10.7202/1028840ar

Giroux, P., Gagnon, M., Gremion, C. et Heinzen, S. (2014). Regard de futurs enseignants sur l'importance des compétences TIC (Internet) pour les jeunes et la responsabilité de divers intervenants à cet égard. Formation et profession, 22(1), 1326. http://dx.doi.org/10.18162/fp.2014.18

Giroux, P., Gagnon, M., Lessard, S. et Cornut, J. (2011). Using Internet information: Undergraduate teachers' critical competencies. Research in Education and Media, 3(1), 123-139.

Hjorland, B. (2012). Methods for evaluating information sources: An annotated catalogue. Journal of Information Science, 38(3), 258-268. http://dx.doi.org/10.1177/0165551512439178

Hogan, N. R. et Varnhagen, C. K. (2012). Critical appraisal of information on the Web in practice: Undergraduate students' knowledge, reported use, and behaviour. Canadian Journal of Learning and Technology, 38(1). http://dx.doi.org/10.21432/T23K5P

International Society for Technology in Education. (2008). ISTE standards for educators. Repéré à http://www.iste.org/standards/ISTE-standards/standards-for-teachers 
Karsenti, T., Dumouchel, G. et Komis, V. (2014). Les compétences informationnelles des étudiants à l'heure du Web 2.0 : proposition d'un modèle pour baliser les formations. Documentation et bibliothèques, 60(1), 20-30. http://dx.doi.org/10.7202/1022859ar

Kuhlthau, C. C. (1993). Seeking meaning: A process approach to library and information services. Norwood, NJ : Ablex.

Larivée, S. (2017). Regards croisés sur l'analphabétisme scientifique et le processus d'évaluation par les pairs. Revue de psychoéducation, 46(1), 1-21. http://dx.doi.org/10.7202/1039679ar

Lefebvre, S. (2014). Intégration des technologies de l'information et de la communication : types de connaissances abordées dans le discours d'enseignants en exercice et d'étudiants en formation initiale. Revue canadienne de l'éducation, 37(3). Repéré à http://journals.sfu.ca/cje/index.php/cje-rce/article/view/1529

Marchionini, G. (1995). Information seeking in electronic environments. Cambridge, MA : Cambridge University Press. http://dx.doi.org/10.1017/cbo9780511626388

Martinet, M. A., Raymond, D. et Gauthier, C. (2001). La formation à l'enseignement : les orientations, les compétences professionnelles. Repéré à http://www.education.gouv.qc.ca/fileadmin/site web/documents/reseau/formation titularisation/formation_enseignement_orientations_EN.pdf

Maubant, P. (2007). L'analyse des pratiques enseignantes : les ambiguïtés d'un bel objet de recherche. Formation et profession, 13(2), 17-21. Repéré à http://www.crifpe.ca/download/verify/123

Metzger, M. J. (2007). Making sense of credibility on the Web: Models for evaluating online information and recommendations for future research. Journal of the American Society for Information Science and Technology, 58(13), 2078-2091. http://dx.doi.org/10.1002/asi.20672

Miles, M. B. et Huberman, A. M. (2003). Analyse des données qualitatives (2éd.). Bruxelles : De Boeck Université.

Ministère de l'Enseignement supérieur et de la Recherche. (2011). Référentiel national du certificat informatique et internet de l'enseignement supérieur de niveau 2 «enseignant ». Bulletin officiel $n^{\circ} 5$ du 3 février 2011. Repéré à https://c2i.education.fr/IMG/pdf/BO 5 03022011_Arrete14122010_Organisation.pdf

Mintz, A. P. (dir.). (2012). Web of deceit: Misinformation and manipulation in the age of social media. Medford, NJ : Information Today.

Peres, E. (2015). Les données numériques : un enjeu d'éducation et de citoyenneté. Paris : Conseil économique, social et environnemental.

Sahut, G. (2014). Les jeunes, leurs enseignants et Wikipédia : représentations en tension autour d'un objet documentaire singulier. Documentaliste-Sciences de l'information, 51(2), 70-79. http://dx.doi.org/10.3917/docsi.512.0070

Serres, A. (2005). Évaluation de l'information sur Internet : Le défi de la formation. Bulletin des bibliothèques de France, (6), 38-44. Repéré à http://bbf.enssib.fr/consulter/bbf-2005-06-0038-006

Serres, A. (2012). Dans le labyrinthe : évaluer l'information sur Internet. Caen : C \& F Éditions.

Shaik, S. (2017). Improvements in protecting the integrity of activity on Facebook. Repéré à https://fr-ca.facebook.com/notes/ facebook-security/improvements-in-protecting-the-integrity-of-activity-on-facebook/10154323366590766

Simard, S. et Karsenti, T. (2016). A quantitative and qualitative inquiry into future teachers' use of information and communications technology to develop students' information literacy skills. Canadian Journal of Learning and Technology, 42(5). http://dx.doi.org/10.21432/T2DW5K

Simonnot, B. (2007). Évaluer l'information. Documentaliste-Sciences de l'information, 44(3), 210-216. http://dx.doi.org/10.3917/docsi.443.0210

Stockham, M. et Collins, H. (2012). Information literacy skills for preservice teachers: Do they transfer to K-12 classrooms? Education Libraries, 35(1-2), 59-72. http://dx.doi.org/10.26443/el.v35i1-2.316

Sutter, E. (1998). Pour une écologie de l'information. Documentaliste-Sciences de l'information, 35(2), 83-86.

Tardif, J. (1998). Intégrer les nouvelles technologies de l'information : quel cadre pédagogique? Paris : ESF. 
Uppal, T. (2011). Internet use for lesson preparation by pre-service teachers: A mixed methods study (Mémoire de maîtrise, University of Toronto). Repéré à https://tspace.library.utoronto.ca/handle/1807/30110

Van der Maren, J.-M. (1996). Méthodes de recherche pour l'éducation (2éd.). Montréal, QC : Presses de l'Université de Montréal.

Watson, C. (2014). An exploratory study of secondary students' judgments of the relevance and reliability of information. Journal of the Association for Information Science and Technology, 65(7), 1385-1408. http://dx.doi.org/10.1002/asi.23067

\section{Pour citer cet article}

Dumouchel, G. et Karsenti, T. (2019). Comment les futurs enseignants du Québec évaluent l'information trouvée sur le Web : une étude des pratiques déclarées et effectives. Formation et profession. 27(2), 74-87.

http://dx.doi.org/10.18162/fp.2019.449 